\title{
Investigation of Water-free Biotissue-mimicking Phantoms in Terahertz Frequency Range
}

Tianmiao Zhang ${ }^{1}$, Mikhail K. Khodzitsky ${ }^{1}$, Petr S. Demchenko ${ }^{1}$, Alexander V. Bykov ${ }^{1,2}$, Alexey P. Popov $^{1,2}$ and Igor V. Meglinski ${ }^{1,2}$

${ }^{1}$ Terahertz Biomedicine Laboratory, ITMO University, Saint Petersburg, 199034 Russia

${ }^{2}$ Optoelectronics and Measurement Techniques Unit, University of Oulu, Oulu, 90014 Finland

\begin{abstract}
:
PVC-based water-free phantoms with silicon and zinc oxide nanoparticles were fabricated for mimicking biotissues in the terahertz frequency range. Terahertz time-domain spectroscopy (TDS) was used to obtain the refractive indices and absorption coefficients of the phantoms. Their optical properties were compared with those of real biotissues from published data. The results show that the phantoms are able to mimic human skin, paraffin-embedded glioma and paraffin-embedded healthy brain tissue by their optical properties. The refractive index of the phantoms can be controlled by changing the concentration of the nanoparticles.
\end{abstract}

\section{Introduction}

Terahertz (THz) spectroscopy application for medical purposes is expanding rapidly due to its safety feature, growing number of global population and development of novel diagnostic technologies [1][2][3][4][5]. Development of any $\mathrm{THz}$ biomedical devices requires phantoms for device testing and calibration. Real biological tissues and organs are not suitable for this purpose because of instability of their properties, in particular, due to presence of water and its evaporation. Since THz radiation is very sensitive to water content [3]-[4],[5], this will significantly affect the measured signal. Therefore, waterfree phantoms are in high demand to substitute real biological tissues and should be developed.

\section{Sample Preparation}

Usually, such phantoms consist of transparent or low scattering matrix/base material, embedded scatterers and absorbers at different concentrations mimicking scattering and absorption properties of real tissues [6]. In this research, we designed and produced two types of phantoms. One type of phantoms was fabricated with zinc oxide $(\mathrm{ZnO})$ nanoparticles as a scattering agent. The concentration of $\mathrm{ZnO}$ was $12 \mathrm{mg} / \mathrm{ml}$. Another type of phantoms was fabricated with silicon ( $\mathrm{Si}$ ) nanoparticles as a scattering agent. The concentrations of Si were $0 \mathrm{mg} / \mathrm{ml}, 0.001 \mathrm{mg} / \mathrm{ml}, 0.01 \mathrm{mg} / \mathrm{ml}$ and $0.1 \mathrm{mg} / \mathrm{ml}$. Polyvinyl chlorideplastisol (PVCP) was used as a matrix material. PVCP and the nanoparticles were then mixed with each other. After sonication for $15 \mathrm{~min}$, the mixture was poured into a rectangular aluminum mold and put into an oven $\left(180^{\circ} \mathrm{C}\right)$ for $30 \mathrm{~min}$. Finally, after cooling, the fabricated phantom was stored between glass slides. Photos of the phantoms is shown in Fig. 1. 

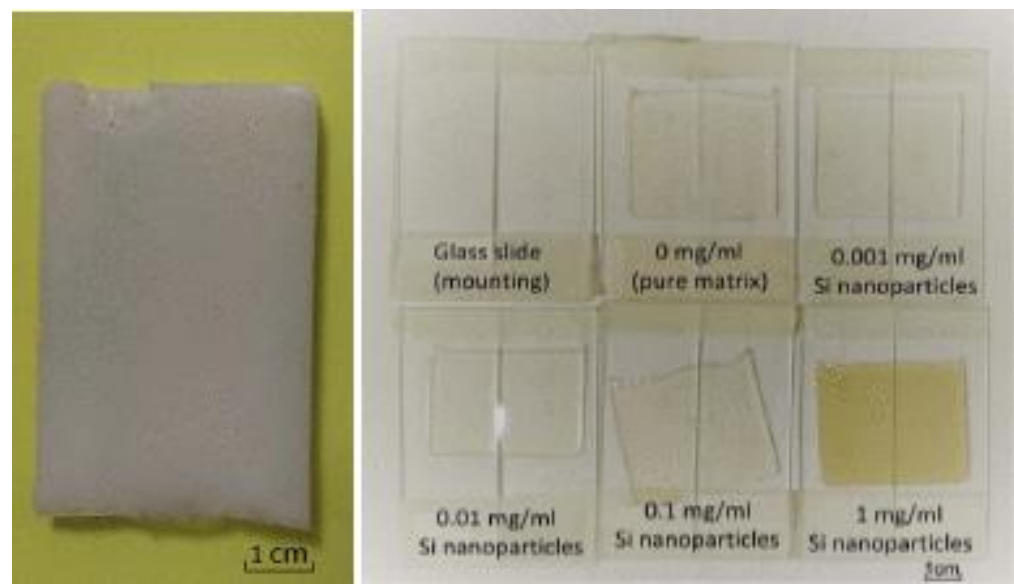

Fig. 1. The fabricated PVC-based water-free phantom with $\mathrm{ZnO}$ nanoparticles (left), glass slides and the fabricated PVC-based water-free phantom with different concentration of Si nanoparticles (right).

\section{Experimental Setup}

The experiment was carried out using a THz time-domain spectroscopy (TDS). The diagram of THz TDS is shown in Fig. 2. The femtosecond infrared laser generates a laser beam in a series of pulses. A beam splitter divides the infrared beam into two beams. One beam travels through a time delay line and then passes the semiconductor material indium arsenide ( $\mathrm{InAs}$ ) to generate $\mathrm{THz}$ radiation. $\mathrm{The} \mathrm{THz}$ radiation then penetrates through the sample and reaches another semiconductor material, cadmium telluride (CdTe). Meanwhile, another beam, known as a probe beam, having travelled through a series of polarizers, finally meets with the $\mathrm{THz}$ beam transmitted through the sample on the CdTe surface. Each pulse of the probe beam interacts with one part of the transmitted $\mathrm{THz}$ pulse. By moving the time delay line, the probe beam can interact with the whole transmitted $\mathrm{THz}$ pulse. By comparing the polarization difference of these two beams using a balanced detector, the transmitted waveform of the sample is finally recorded. Our THz TDS works in the frequency range of $0.1-1 \mathrm{THz}$.

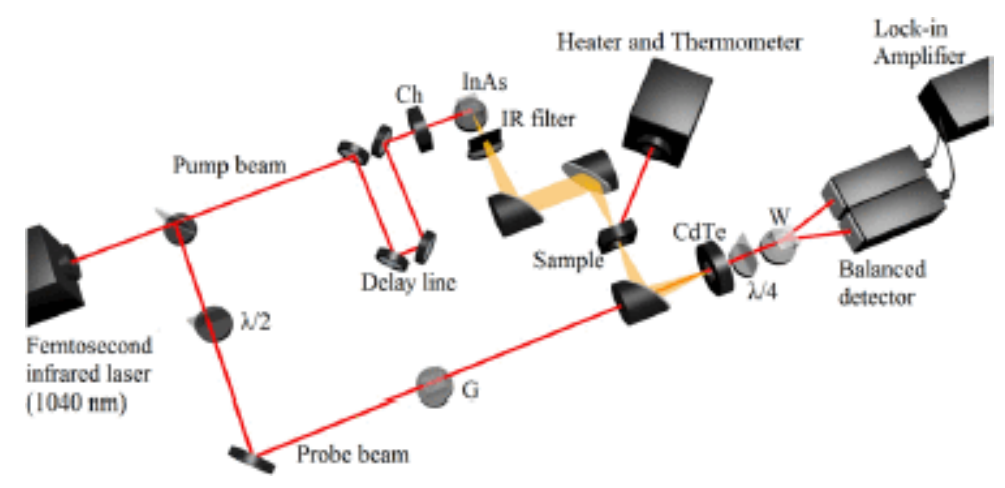

Fig. 2. The diagram of thz TDS system. $\lambda / 2$ - half-wave plate, $G$ - Glan prism, $C h$ - chopper, $\lambda / 4$ - quarterwave plate, $\mathrm{W}$ - wollaston prism.

\section{Signal Processing}

To obtain more reliable result, we used wavelet to process the signals. We chose Daubechies 6 (db6) wavelet and 'SqTwoLog' thresholding technique as a filtration complex [7]. Mallat algorithm was chosen as a filtration algorithm. The basic formulas of the Mallat algorithm are shown below [8]. The signal is decomposed using formula (1), then thresholding technique is applied. 


$$
\begin{gathered}
\left\{\begin{array}{c}
W_{2^{j+1}} f(t)=\frac{1}{\lambda_{j}} S_{2^{j}} f(t) * G_{j} \\
S_{2^{j+1}} f(t)=S_{2^{j}} f(t) * H_{j}
\end{array}\right. \\
S_{2^{j-1}} f=\lambda_{j} W_{2^{j}} f * K_{j-1}+S_{2^{j}} f * H_{j-1}
\end{gathered}
$$

where $\mathrm{Gj}, \mathrm{Hj}, \mathrm{Kj}$ are filter bank matrixes and $\lambda \mathrm{j}$ is normalization coefficients [8], [9].

After the filtration, the Fast Fourier Transform (FFT) was applied to obtain spectra and phases of the signals for later analysis. The following formulas were used to calculate the refractive index and absorption coefficient based on the spectra and the phases obtained [10]:

$$
\begin{gathered}
\alpha(v)=-2 \ln \left[\frac{T(v) E_{\text {sample }}(v)}{E_{\text {reference }}(v)}\right] / d \\
T(v)=1-R=1-[n(v)-1]^{2} /[n(v)+1]^{2} \\
n(v)=1+c\left[\phi_{\text {sample }}(v)-\phi_{\text {reference }(v)}\right] /[2 \pi v d]
\end{gathered}
$$

where $\alpha$ is the absorption coefficient, $\mathrm{n}$ is the refractive index of the sample, $\mathrm{E}$ and $\phi$ are the amplitude and the phase of the signal, $\mathrm{d}$ is the thickness of the sample, $\mathrm{R}$ is the Fresnel loss at the substrate-sample interface.

\section{Results}

After application of formulas (4) and (5), the refractive index and absorption coefficients of the phantoms were obtained and were compared with the published data [11], [12].

Fig. 3 shows that the phantom with $\mathrm{ZnO}$ nanoparticles is able to mimic the refractive index of human hand skin in the frequency range of $0.4-0.65 \mathrm{THz}$, since the refractive indices of the phantom and hand skin are very close to each other. The absorption coefficients are significantly lower than that of kin due to its water-free nature.
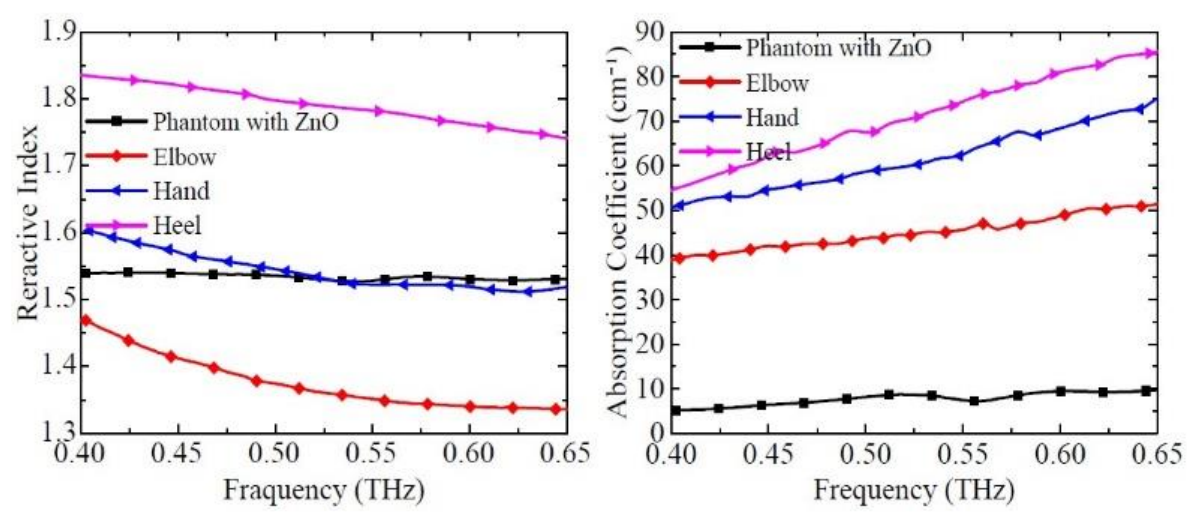

Fig. 3. Comparison of the refractive indices and the absorption coefficients of phantom with $\mathrm{ZnO}$ nanoparticles, elbow, hand and heel skin. The data of elbow, hand and heel skin are from article [11]. 

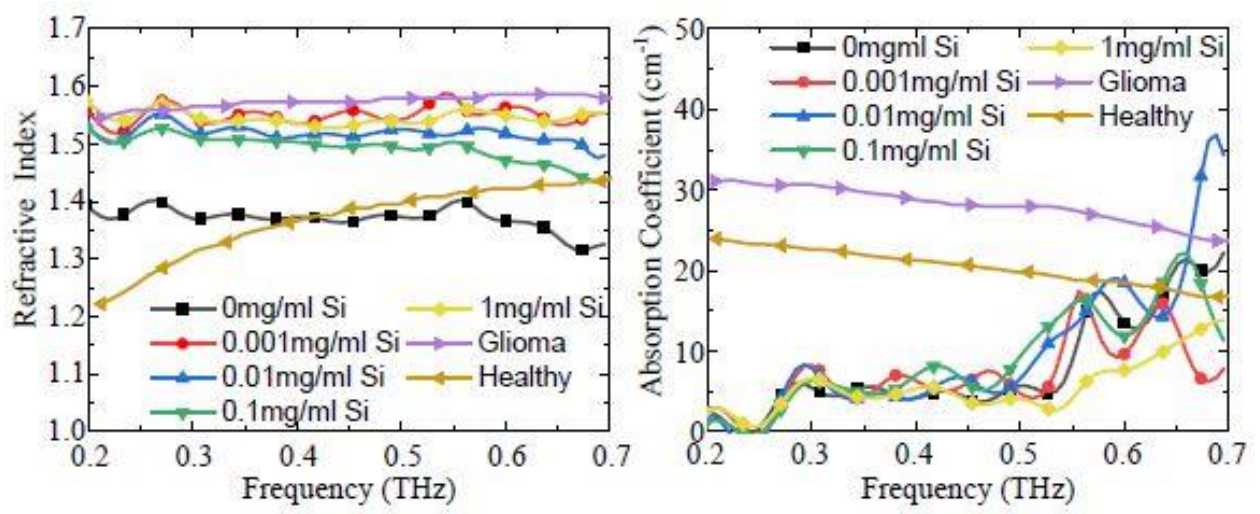

Fig. 4. Comparison of the refractive indices and the absorption coefficients of phantoms with $\mathrm{Si}$ nanoparticles, paraffin-embedded glioma and paraffin embedded healthy tissue. The data of glioma and healthy tissue is from article [12].

The phantoms with Si nanoparticles (Fig. 4) mimic the paraffin-embedded brain glioma by its refractive index in the frequency range of $0.2-0.7 \mathrm{THz}$, especially when the concentration of Si nanoparticles is $0.001 \mathrm{mg} / \mathrm{ml}$. The results also show that the phantom can be controlled to obtain different refractive index by changing the concentration of nanoparticles. Moreover, the pure PVC matrix can mimic healthy brain tissue by its refractive index. The most suitable frequency range the phantom application is $0.35-0.55$ $\mathrm{THz}$, due to the fact that the refractive index of our phantom is nearly invariable, while the refractive index of healthy brain tissue slightly increases along with the frequency. The absorption coefficients are lower than glioma and healthy tissue in the frequency range of $0.2-0.5 \mathrm{THz}$.

\section{Summary}

The results prove that the presented phantoms with $\mathrm{ZnO}$ nanoparticles, Si nanoparticles and the phantom with no nanoparticles can mimic human hand skin, paraffin-embedded glioma and paraffin-embedded healthy brain tissue, respectively, in THz frequency range. The influence of different concentration of nanoparticles on the refractive indices of the phantoms are also revealed. It gives a good promise that by choosing proper concentrations we may mimic the optical properties of other bio-tissues. Because of the water-free characteristic of the phantoms, the absorption coefficients of all phantoms are lower than the absorption coefficients of those biotissues.

\section{Acknowledgements}

This work was financially supported by the Government of the Russian Federation, Grant 08-08. AB and AP acknowledge the Academy of Finland (Grants 290596 and 314369).

\section{References}

[1] M. V. Duka, L. N. Dvoretskaya, et al. "Numerical and experimental studies of mechanisms underlying the effect of pulsed broadband terahertz radiation on nerve cells," Quantum Electron, vol. 44, no. 8, pp. 707-712, 2014.

[2] M. Borovkova, M. Serebriakova, et al., "Investigation of terahertz radiation influence on rat glial cells," Biomed. Opt. Express, vol. 8, no. 1, pp. 273-280, 2017.

[3] M. Borovkova, M. Khodzitsky, et al. "Terahertz time-domain spectroscopy for noninvasive assessment of water content in biological samples," Biomed.Opt. Express, vol. 9, no. 5, pp. 2266-2276, 2018.

[4] S. I. Gusev, P. S. Demchenko, et al., "Influence of glucose concentration on blood optical properties in THz frequency range," Chinese Optics, vol. 11, no. 2, pp. 182-189, 2018. 
[5] O. A. Smolyanskaya, O. V. Kravtsenyuk, et al. "Study of blood plasma optical properties in mice grafted with Ehrlich carcinoma in the frequency range 0.1-1.0 THz," Quantum Electron, vol. 47, no. 11, pp. 1031, 2017.

[6] M. Wrobel, et al. "Nanoparticle-free tissue-mimicking phantoms with intrinsic scattering," Biomed. Opt. Express, vol. 7, no. 6, pp. 2088-2094, 2016.

[7] J. Pei, P. Ye, and W. Xie, "Optimal wavelet analysis for THz-TDS pulse signals," Proc. SPIE, no. 7277, pp. 727708, 2008.

[8] S. Mallat, and S. Zhang, "Characterization of signals from multiscale edges," IEEE Trans. Pattern Anal. Mach. Intel, vol.14, no. 7, pp. 710-732,

1992.

[9] G. Strang, and T. Nguyen, Wavelets and Filter Banks. Wellesley, MA: Wellesley-Cambridge Press, 1996.

[10] H. Yasuda, "Measurement of terahertz refractive index of metal with terahertz time-domain spectroscopy," Jpn. J. Appl. Phys, vol. 47, no. 3R, pp. 1632-1634, 2008.

[11] K. I. Zaytsev, et al. "Highly accurate in vivo terahertz spectroscopy of healthy skin: variation of refractive index and absorption coefficient along the human body," IEEE Trans. THz Sci. Technol, vol. 5, no. 5, pp. 817-827, 2015.

[12] K. Meng, et al. "Terahertz pulsed spectroscopy of paraffin- embedded brain glioma," J. Biomed. Opt, vol. 19, no. 7, pp. 077001, 2014. 